\title{
A Single-Center, Retrospective Study of Bevacizumab-Containing Neoadjuvant Chemotherapy followed by Interval Debulking Surgery for Ovarian Cancer
}

\author{
Junsik Park, Kyung Jin Eoh, Eun Ji Nam, Sunghoon Kim, Sang Wun Kim, Young Tae Kim, and Jung-Yun Lee \\ Department of Obstetrics and Gynecology, Institute of Women's Life Medical Science, Yonsei University College of Medicine, Seoul, Korea.
}

\begin{abstract}
Purpose: We evaluated whether adding bevacizumab to current platinum-based chemotherapy could improve clinical outcomes without affecting safety.

Materials and Methods: We retrospectively reviewed medical records of patients with pathologically confirmed ovarian cancer who received neoadjuvant chemotherapy (NAC) at Yonsei Cancer Hospital. We divided the patients into groups based on the use of bevacizumab for NAC (CP group: carboplatin+paclitaxel vs. BCP group: bevacizumab+carboplatin+paclitaxel) and compared patient characteristics, responses to NAC, and surgical and survival outcomes between the two groups. Overall, 88 patients in the $\mathrm{CP}$ group and 16 patients in the BCP group received NAC. The primary endpoint was survival outcomes. Complete resection rate after interval debulking surgery (IDS), cancer antigen 125 (CA-125) normalization after NAC, and chemotherapy response score were secondary endpoints.

Results: After NAC treatment, all patients underwent IDS. There were no significant differences in adverse events during NAC or postoperative complications between the two groups ( $p=0.293$ and $p=0.485$, respectively). There were also no significant differences in CA-125 normalization after NAC ( $42.0 \%$ vs. $43.8 \%, p=0.899)$ or complete resection rate after IDS ( $47.7 \%$ vs. $56.3 \%, p=0.530)$. However, although the BCP group did not show longer overall survival (OS) (log-rank $p=0.854)$, they had significantly longer progression-free survival (PFS) than the CP group (log-rank $p=0.048$ ).

Conclusion: Bevacizumab-containing NAC might be safe and provide longer PFS than chemotherapy alone in patients with advanced ovarian cancer. However, further study is necessary to investigate the impact of bevacizumab-containing NAC on OS.
\end{abstract}

Key Words: Bevacizumab, epithelial ovarian cancer, interval debulking surgery, neoadjuvant chemotherapy

\section{INTRODUCTION}

Primary debulking surgery followed by adjuvant chemothera-

Received: December 11, 2019 Revised: March 3, 2020

Accepted: March 4, 2020

Corresponding author: Jung-Yun Lee, MD, PhD, Department of Obstetrics and Gynecology, Institution of Women's Medical Life Science, Yonsei University College of Medicine, 50-1 Yonsei-ro, Seodaemun-gu, Seoul 03722, Korea.

Tel: 82-2-2228-2237, Fax: 82-2-313-8357, E-mail: jungyunlee@yuhs.ac

-The authors have no potential conflicts of interest to disclose.

(C) Copyright: Yonsei University College of Medicine 2020

This is an Open Access article distributed under the terms of the Creative Commons Attribution Non-Commercial License (https://creativecommons.org/licenses/ by-nc/4.0) which permits unrestricted non-commercial use, distribution, and reproduction in any medium, provided the original work is properly cited. py is considered a standard treatment for ovarian cancer. However, for patients with advanced ovarian cancer and with a high perioperative risk or a low likelihood of achieving optimal cytoreduction, neoadjuvant chemotherapy (NAC) followed by interval debulking surgery (IDS) may be an alternative treatment option. ${ }^{1-4}$ After the ICON7 and GOG-218 trials, bevacizumab, a humanized VEGF-neutralizing monoclonal antibody, was proposed as an additive to first-line ovarian cancer therapies: the ICON7 and GOG-218 trials showed that bevacizumab improved progression-free survival (PFS) when incorporated with platinum-based adjuvant chemotherapy., ${ }^{5,6}$ According to the ANTHALYA trial, bevacizumab-containing NAC is safe and increases the complete resection rate after IDS. ${ }^{7}$ In recent years, the number of patients with advanced ovarian 
cancer treated with this NAC-IDS strategy has increased. However, data are scarce on the efficacy and safety of NAC regimens that incorporate bevacizumab. In the ANTHALYA trial, $15 \mathrm{mg} / \mathrm{kg}$ of bevacizumab was added to the NAC regimen; however, $7.5 \mathrm{mg} / \mathrm{kg}$ of bevacizumab (the dose used in ICON7) is the current dose permitted in Korea by the Health Insurance Review \& Assessment Service.

In this study, we retrospectively investigated the efficacy and safety of a bevacizumab-containing NAC regimen followed by IDS in patients with newly diagnosed advanced ovarian cancer in Korea. We hypothesized that NAC with bevacizumab could be safely administered to patients with advanced ovarian cancer and would lead to more successful IDS, thereby improving survival outcomes.

\section{MATERIALS AND METHODS}

\section{Study population and patient characteristics}

We retrospectively reviewed the medical records of 112 patients with pathologically confirmed epithelial ovarian cancer who received at least one cycle of NAC at Yonsei Cancer Hospital between August 2016 and May 2019. All patients were histologically or cytologically confirmed to have International Federation of Gynecology and Obstetrics (FIGO) stage IIIC-IV epithelial ovarian cancer before starting chemotherapy. Diagnoses were obtained via either laparoscopic or image-biopsy samples or fine-needle aspiration of a tumor site or ascites/ effusion. All surgical procedures were performed by one of five gynecologic oncology surgeons at our institute. Histological diagnoses were based on World Health Organization criteria, and all microscopic slides were reviewed by two experienced gynecologic pathologists.

NAC was performed if at least one of the following three criteria was met: ${ }^{8}$ 1) pulmonary and/or hepatic parenchymal metastases were observed in imaging studies before surgery, 2) the patient was medically inoperable, or 3) optimal cytoreduction was not achievable owing to a high tumor burden (Fagotti score $\geq 8$ ) observed by diagnostic laparoscopy. ${ }^{9,10}$ According to our institutional policy, IDS was performed after three cycles of NAC. The timing of IDS was delayed when optimal cytoreduction was not achievable, as determined at the clinician's discretion.

We excluded patients who had other malignancies $(n=1)$, who had been treated with immune checkpoint inhibitors $(n=3)$, or who had incomplete medical records owing to loss at followup $(n=4)$. The final study population comprised 104 patients (Fig. 1). Patients were divided into two groups based on the administration of bevacizumab with NAC.

The following data were extracted from the patients' medical records: age, body mass index (BMI), American Society of Anesthesiologists (ASA) score, serum cancer antigen 125 (CA125) levels, serum human epididymis protein 4 (HE4) levels, FIGO stage, histology, residual disease after IDS, date of progression or recurrence, and date of last follow up. The present study was reviewed and approved by our Institutional Review Board (4-2018-0518) in accordance with the Declaration of Helsinki and the International Conference on Harmonisation Good Clinical Practice guidelines.

\section{NAC, surgery, and perioperative complications}

All NAC regimens included carboplatin and paclitaxel (CP).

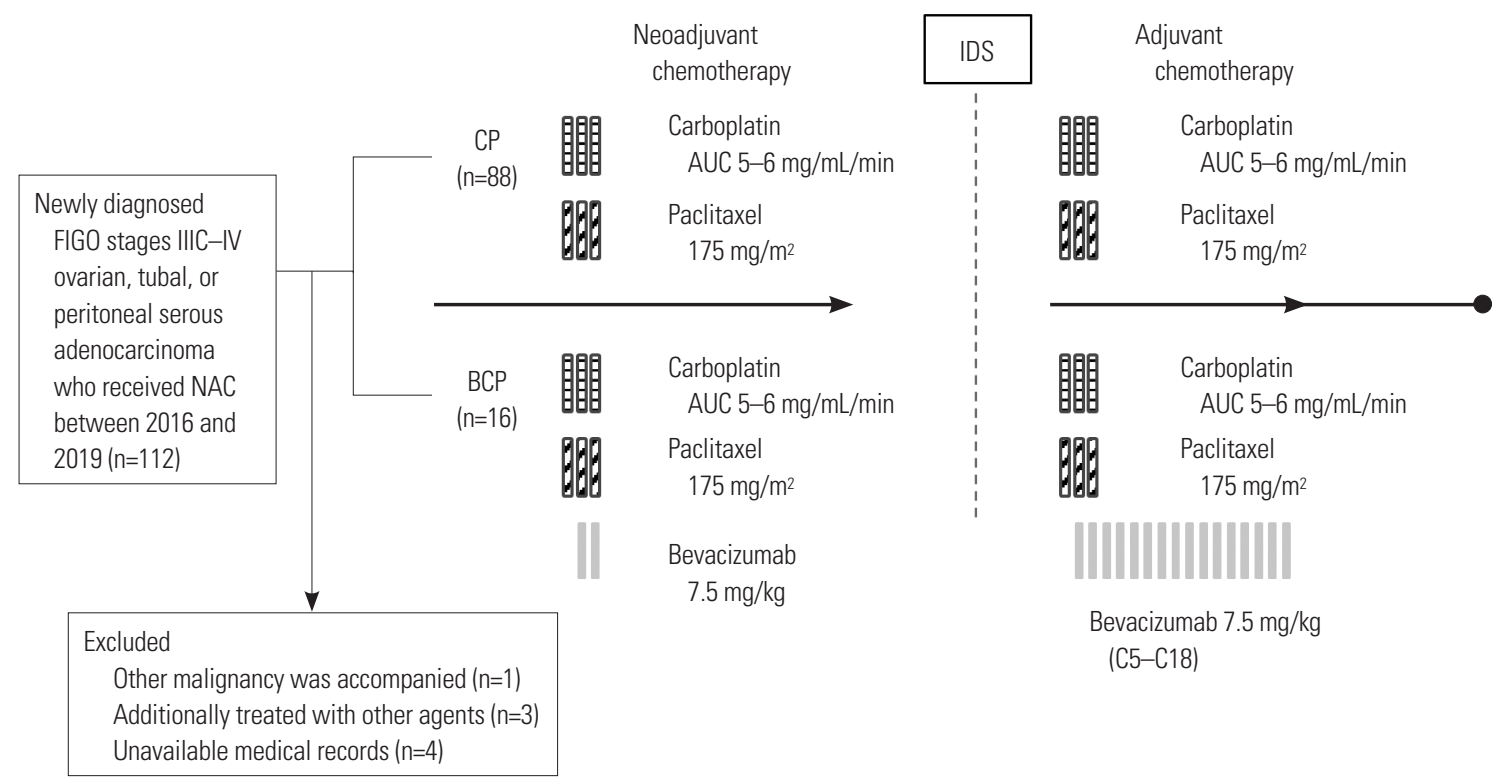

Fig. 1. Flow diagram of the study population consisting of the standard carboplatin-paclitaxel chemotherapy group (CP, $n=88$ ) and the bevacizumab-containing chemotherapy group (BCP, n=16). FIGO, Federation of Gynecology and Obstetrics; NAC, neoadjuvant chemotherapy; IDS, interval debulking surgery; AUC, area under the curve. 
In addition, stage IIIC or IV patients for whom optimal primary cytoreduction was not achievable, owing to a high tumor burden, additionally received bevacizumab (BCP). Because the study was retrospective, use of the bevacizumab was determined in accordance with the clinician's discretion, and 16 patients were treated with BCP. Dosing schedules for NAC were as follows: all patients received paclitaxel $\left(175 \mathrm{mg} / \mathrm{m}^{2}\right)$ and carboplatin (area under the curve, $5-6 \mathrm{mg} / \mathrm{mL} / \mathrm{min}$ ) every 3 weeks for cycles $1-3$. Patients in the BCP group additionally received bevacizumab $(7.5 \mathrm{mg} / \mathrm{kg})$ every 3 weeks in cycles 2 and 3. Conventional surgical procedures included the sampling of free fluid or peritoneal washings for cytology; a thorough inspection of the abdomen and pelvis, including the upper abdominal viscera, diaphragm, and retroperitoneal spaces; and hysterectomy, bilateral oophorectomy and omentectomy, pelvic/para-aortic lymph node dissection, and appendectomy. Radical surgery included bowel resection, diaphragm or other peritoneal surface stripping, splenectomy, partial hepatectomy, partial gastrectomy, partial cystectomy and/or ureteroneocystostomy, cholecystectomy, and/or distal pancreatectomy. ${ }^{11-13}$

Perioperative complications were graded according to the Memorial Sloan-Kettering Cancer Center surgical secondary events grading system; $;^{14,15}$ a score $\geq 3$ indicated a major complication. Operative mortality was defined as death occurring within 30 days after surgery (grade 5).

\section{Surveillance and follow-up}

We routinely evaluated CA-125 levels and performed imaging studies for surveillance. Our institutional follow-up strategy was to follow up patients every 3 months for the first 2 years after treatment and every 6 months thereafter. Recurrence was defined as the date of appearance of radiologically detected disease during a follow-up examination. An increase in CA125 levels without clinical signs of relapse was not considered to indicate progression, but prompted further radiological examinations. PFS was defined as the time from diagnosis to objective disease progression on imaging (according to modified response evaluation criteria in solid tumor (RECSIST), version 1.1).

\section{Statistical analysis}

The Statistical Package for Social Sciences software (version 21.0; IBM Corp., Armonk, NY, USA) was used for all statistical analyses. Descriptive statistics were used for demographic data and are summarized as medians (ranges) or frequencies (percentages). Differences in patient characteristics between groups were compared with respect to time intervals using chisquare or Mann-Whitney U tests. Overall survival (OS) and PFS were analyzed using the Kaplan-Meier method and logrank tests. For all analyses, $p<0.05$ indicated statistical significance.

\section{RESULTS}

\section{Patient demographics}

Of 112 patients, 104 were eligible for analysis in this study. Of these, 88 patients received conventional NAC (CP), and 16 patients received bevacizumab-containing NAC (BCP). Patient and clinical characteristics are shown in Table 1 . The median ages, mean BMI, and ASA scores of patients in both groups were comparable. Although the serum CA-125 and HE4 levels and FIGO stages at diagnosis of the BCP group were higher

Table 1. Baseline and Disease Characteristics

\begin{tabular}{|c|c|c|c|c|}
\hline & $\begin{array}{c}C P \\
(n=88)\end{array}$ & $\begin{array}{c}\text { BCP } \\
(n=16)\end{array}$ & $\begin{array}{c}\text { Total } \\
(\mathrm{n}=104)\end{array}$ & $p$ value \\
\hline \multicolumn{5}{|l|}{ Age (yr) } \\
\hline Median (range) & $58(39-77)$ & 56 (39-78) & $58(39-78)$ & 0.943 \\
\hline $\mathrm{BMI}, \mathrm{kg} / \mathrm{m}^{2}$ & $23.67 \pm 3.61$ & $23.38 \pm 4.24$ & $23.62 \pm 3.69$ & 0.702 \\
\hline FIGO stage & & & & 0.164 \\
\hline IIIC & $37(42.0)$ & $3(18.8)$ & 40 (38.5) & \\
\hline IVA & $7(8.0)$ & $3(18.8)$ & $10(9.6)$ & \\
\hline IVB & $44(50.0)$ & $10(62.5)$ & $54(51.9)$ & \\
\hline Histologic type & & & & 0.353 \\
\hline HGSC & 80 (81.2) & $16(100)$ & $96(92.3)$ & \\
\hline Non-HGSC & $8(9.1)$ & $0(0.0)$ & $8(7.7)$ & \\
\hline Histologic grade & & & & 0.353 \\
\hline G1 & $3(3.4)$ & $0(0.0)$ & $3(2.9)$ & \\
\hline G2 & $2(2.3)$ & $0(0.0)$ & $2(1.9)$ & \\
\hline G3 & $83(94.3)$ & $16(100)$ & 99 (95.2) & \\
\hline Germline BRCA statu & & & & 0.873 \\
\hline Wild & $51(63.7)$ & $10(66.7)$ & $61(64.2)$ & \\
\hline Mutation & $23(28.7)$ & $3(20.0)$ & $26(27.4)$ & \\
\hline VOUS & $6(7.5)$ & $2(25.0)$ & $8(8.4)$ & \\
\hline Not available* & 8 & 1 & 9 & \\
\hline $\begin{array}{l}\text { CA-125 level at } \\
\text { diagnosis, U/mL }\end{array}$ & $\begin{array}{c}2727.73 \pm \\
3848.45\end{array}$ & $\begin{array}{c}4600.20 \pm \\
4823.63\end{array}$ & $\begin{array}{c}3015.81 \pm \\
4044.65\end{array}$ & 0.077 \\
\hline$\leq 500 \mathrm{U} / \mathrm{mL}$ & $23(26.1)$ & $4(25.0)$ & $27(26.0)$ & $>0.999$ \\
\hline$>500 \mathrm{U} / \mathrm{mL}$ & 65 (73.9) & $12(75.0)$ & $77(74.0)$ & \\
\hline $\begin{array}{l}\text { HE4 level at } \\
\text { diagnosis }{ }^{\dagger}, \text { pmol/L }\end{array}$ & $\begin{array}{c}629.98 \pm \\
501.73\end{array}$ & $\begin{array}{c}822.79 \pm \\
529.22\end{array}$ & $\begin{array}{c}667.14 \pm \\
509.61\end{array}$ & 0.124 \\
\hline ASA score & & & & 0.444 \\
\hline 1 & $2(2.3)$ & $0(0.0)$ & $2(1.9)$ & \\
\hline 2 & $47(53.4)$ & $7(43.8)$ & $54(51.9)$ & \\
\hline 3 & $37(42.0)$ & $9(56.3)$ & 46 (44.2) & \\
\hline 4 & $2(2.3)$ & $0(0.0)$ & $2(1.9)$ & \\
\hline
\end{tabular}

CP, carboplatin+paclitaxel; BCP, bevacizumab+carboplatin+paclitaxel; BMI, body mass index; FIGO, International Federation of Gynecology and Obstetrics; HGSC, high-grade serous adenocarcinoma; BRCA, breast cancer susceptibility gene; VOUS, variant of unknown significance; CA-125, cancer antigen 125; HE4, human epididymis protein 4; ASA, American Society of Anes-thesiologists.

Values are presented as mean \pm standard deviation or $\mathrm{n}(\%)$ unless otherwise noticed.

*Missing values are excluded from the calculation of percentages, 'The HE4 levels of five patients were not measured at diagnosis. 
than those of the CP group, these differences were not significant. Serous carcinoma histology types were found in over $90 \%$ of all patients. BRCA1 or BRCA2 mutation statuses were comparable between both groups.

\section{Feasibility and safety of bevacizumab-containing NAC followed by interval debulking surgery}

The median number of NAC cycles was three. The frequencies of grade $\geq 3$ adverse events during NAC and perioperative complications within 30 days after IDS are summarized in Table 2. All patients underwent IDS after NAC. Among 104 patients, 7 (5.8\%) experienced major adverse events during NAC. Of these, febrile neutropenia and anemia were the most frequent in both groups. One case of gastrointestinal perforation occurred in the BCP group, but this result was not significant. None of the patients developed grade $\geq 4$ adverse events.

Regarding perioperative complications after IDS, none of the patients developed grade $\geq 4$ adverse events, and the complications are summarized in Table 2. Nineteen (18.3\%) patients experienced perioperative complications within 30 days after IDS. Among these complications, blood transfusion was the most common in the BCP group (18.8\%); however, this was not statistically significant. In addition, none of the patients developed bleeding complications, thromboembolic complications, fistulas/abscesses, or perforations in the BCP group.

Table 2. Adverse Events During NAC and Perioperative Complications after IDS

\begin{tabular}{|c|c|c|c|c|}
\hline & $\begin{array}{c}\text { CP } \\
(n=88)\end{array}$ & $\begin{array}{c}\text { BCP } \\
(n=16)\end{array}$ & $\begin{array}{c}\text { Total } \\
(\mathrm{n}=104)\end{array}$ & $p$ value \\
\hline \multicolumn{4}{|c|}{ Adverse events grade $\geq 3$ during NAC } & 0.293 \\
\hline Adverse events & $5(5.7)$ & $2(12.5)$ & $7(5.8)$ & \\
\hline Febrile neutropenia & $3(3.4)$ & $1(6.3)$ & $4(3.8)$ & \\
\hline Anemia & $2(2.3)$ & $0(0.0)$ & $2(1.9)$ & \\
\hline Thrombocytopenia & $0(0.0)$ & $0(0.0)$ & $0(0.0)$ & \\
\hline Fatigue & $0(0.0)$ & $0(0.0)$ & $0(0.0)$ & \\
\hline Gastrointestinal $^{*}$ & $0(0.0)$ & $1(6.3)$ & $1(1.0)$ & \\
\hline Nausea & $0(0.0)$ & $0(0.0)$ & $0(0.0)$ & \\
\hline Infection & $0(0.0)$ & $0(0.0)$ & $0(0.0)$ & \\
\hline Thrombosis & $0(0.0)$ & $0(0.0)$ & $0(0.0)$ & \\
\hline Peripheral neuropathy & $0(0.0)$ & $0(0.0)$ & $0(0.0)$ & \\
\hline \multicolumn{4}{|c|}{ Perioperative complication $\geq$ grade 3 up to 30 days after IDS } & 0.485 \\
\hline Complications & $15(17.0)$ & $4(25.0)$ & $19(18.3)$ & \\
\hline Wound complications $^{\dagger}$ & $2(2.3)$ & $1(6.3)$ & $3(2.9)$ & \\
\hline Infection & $6(6.8)$ & $0(0.0)$ & $6(5.8)$ & \\
\hline Gastrointestinal $^{\ddagger}$ & $4(4.5)$ & $0(0.0)$ & $4(3.8)$ & \\
\hline Lymphocele & $1(1.1)$ & $0(0.0)$ & $1(1.0)$ & \\
\hline Thromboembolic & $2(2.3)$ & $0(0.0)$ & $2(1.9)$ & \\
\hline Blood transfusion & $0(0.0)$ & $3(18.8)$ & $3(2.9)$ & \\
\hline
\end{tabular}

CP, carboplatin+paclitaxel; BCP, bevacizumab+carboplatin+paclitaxel; NAC, neoadjuvant chemotherapy; IDS, interval debulking surgery.

Values are presented as $n(\%)$.

${ }^{*}$ Colonic perforation, ${ }^{\dagger}$ Wound dehiscence, ${ }^{\ddagger}$ Ileus (3), small bowel obstruction (1).

\section{Response to NAC and surgical treatment}

The responses following NAC are summarized in Table 3. In terms of pathological response, omental chemotherapy response scores (CRSs) were reported for 97 of 104 patients (93.3\%). The two groups had comparable omental CRS: a score of 3 was observed in 4 patients $(25.0 \%)$ in the BCP group and 22 patients (27.2\%) in the CP group ( $p=0.736$ ). There were no significant differences in the normalization of serum CA-125 and HE4 levels after NAC between the two groups ( $p=0.899$ and $p=0.453$, respectively).

After NAC treatment, all patients underwent IDS. The results of IDS are shown in Table 4. Regarding types of IDS, most patients $(n=76,86.5 \%)$ underwent open surgery $(86.4 \%$ vs. $87.5 \%$ of the CP and BCP groups, respectively, $p=1.000$ ). Complete resection rates after IDS (R0 state) were not significantly different between the two groups ( $47.7 \%$ vs. $56.3 \%$, respectively, $p=0.530$ ).

At the time of analysis, 13 patients $(11.2 \%$, excluding 4 patients lost in follow up) died, and 54 (45.0\%) experienced re-

Table 3. Responses to NAC Evaluated by Blood Tests, Image Studies, Debulking Status, and CRS

\begin{tabular}{|c|c|c|c|c|}
\hline & $\underset{(n=88)}{C P}$ & $\begin{array}{c}\text { BCP } \\
(n=16)\end{array}$ & $\begin{array}{c}\text { Total } \\
(n=104)\end{array}$ & $\begin{array}{c}p \\
\text { value }\end{array}$ \\
\hline CRS & & & & 0.736 \\
\hline 1 & $3(3.7)$ & $1(6.3)$ & $4(4.1)$ & \\
\hline 2 & $56(69.1)$ & $11(68.8)$ & $67(69.1)$ & \\
\hline 3 & $22(27.2)$ & $4(25.0)$ & $26(26.8)$ & \\
\hline Not available* & 7 & 0 & 7 & \\
\hline Response to NAC & & & & 0.929 \\
\hline $\mathrm{CR}$ & $0(0.0)$ & $0(0.0)$ & $0(0.0)$ & \\
\hline PR & $83(94.3)$ & $15(93.8)$ & $98(94.2)$ & \\
\hline SD & $5(5.7)$ & $1(6.3)$ & $6(5.8)$ & \\
\hline PD & $0(0.0)$ & $0(0.0)$ & $0(0.0)$ & \\
\hline $\begin{array}{l}\mathrm{CA}-125 \text { after NAC, } \\
\mathrm{U} / \mathrm{mL} \text { (range) }\end{array}$ & $\begin{array}{l}281.06 \pm \\
1663.09\end{array}$ & $\begin{array}{c}136.77 \pm \\
194.79\end{array}$ & $\begin{array}{r}258.86 \pm \\
1531.17\end{array}$ & 0.248 \\
\hline \multicolumn{4}{|c|}{ CA-125 normalization after NAC } & 0.899 \\
\hline Normal & $37(42.0)$ & $7(43.8)$ & $44(42.3)$ & \\
\hline Abnormal & $51(58.0)$ & $9(56.3)$ & $60(57.7)$ & \\
\hline $\begin{array}{l}\text { HE4 after NAC }{ }^{\dagger}, \\
\text { pmol/L }\end{array}$ & $121.31 \pm 125.19$ & $133.15 \pm 84.43$ & $124.69 \pm 114.29$ & 0.140 \\
\hline \multicolumn{4}{|c|}{ HE4 normalization after $\mathrm{NAC}^{\dagger}$} & 0.453 \\
\hline Normal & $14(40.0)$ & $4(28.6)$ & $18(36.7)$ & \\
\hline Abnormal & $21(60.0)$ & $10(71.4)$ & $31(63.6)$ & \\
\hline
\end{tabular}

NAC, neoadjuvant chemotherapy; $C$, carboplatin+paclitaxel; BCP, bevacizum ab+carboplatin+paclitaxel; CRS, Chemotherapy Response Score; CR, complete remission; PR, partial remission; SD, stable disease; PD, progressive disease; CA-125, cancer antigen 125; HE4, human epididymis protein 4.

Values are presented as mean \pm standard deviation or $\mathrm{n}(\%)$ unless otherwise noticed.

*The CRS of 39 patients were not measured after NAC. Missing values are excluded from the calculation of percentages, ${ }^{\dagger}$ The HE4 levels of 39 patients were not measured after NAC. Missing values are excluded from the calculation of percentages. 
Table 4. Surgical Details and Results of IDS Evaluated by Blood Tests and Complete Resection Rates

\begin{tabular}{|c|c|c|c|c|}
\hline & $\underset{(n=88)}{C P}$ & $\begin{array}{c}\text { BCP } \\
(n=16)\end{array}$ & $\begin{array}{c}\text { Total } \\
(n=104)\end{array}$ & $p$ value \\
\hline IDS type & & & & 1.000 \\
\hline Open & $76(86.4)$ & $14(87.5)$ & 90 (86.5) & \\
\hline Laparoscopy & $12(11.5)$ & $2(12.5)$ & $14(11.7)$ & \\
\hline Residual disease & & & & 0.530 \\
\hline None (R0) & $42(47.7)$ & $9(56.3)$ & $51(49.0)$ & \\
\hline Any residual & $46(52.3)$ & $7(43.7)$ & $53(51.0)$ & \\
\hline \multicolumn{2}{|c|}{ Interval from IDS to POAC, days } & & & 0.002 \\
\hline Median (range) & $16(7-37)$ & $21.5(10-50)$ & $17(7-50)$ & \\
\hline
\end{tabular}

currence. The relative frequency of patients with recurrence was significantly lower in the BCP group than in the CP group ( $18.8 \%$ vs. $49.0 \%$, respectively; $p=0.023$ ). PFS and OS were analyzed using Kaplan-Meier curves and log-rank tests; the BCP group had longer PFS than the CP group (hazard ratio $=0.32$, 95\% confidence interval 0.22-0.99; log-rank $p=0.048$ ) (Fig. 2A). However, owing to the small sample size and short follow-up period, there was no significant difference in OS between the two groups (hazard ratio $=0.82$, $95 \%$ confidence interval 0.12 5.8; $\log$-rank $p=0.854$ ) (Fig. 2B).

\section{DISCUSSION}

In this study, we investigated the feasibility, safety, and effectiveness of bevacizumab-containing NAC followed by IDS for advanced epithelial ovarian cancer. Our results indicated that bevacizumab could be feasibly and safely added to NAC-IDS regimens for advanced ovarian cancer. Despite other trials that incorporated bevacizumab in NAC, including the ANTHALYA and GEICO 1250 trials, at a standard dose of $15 \mathrm{mg} /$ $\mathrm{kg}$, our institution was limited to using a half dose of bevacizumab $(7.5 \mathrm{mg} / \mathrm{kg}$ ), owing to regulations from the Korean national health system. Although the number of patients treated with bevacizumab in this study was small and the follow-up period was short, we observed improved PFS in patients who received bevacizumab-containing NAC-IDS with safety endpoints comparable to those in patients who received conventional NAC. The current study showed that, at least, bevacizumab-containing NAC-IDS could be safely applied in real practice and could show effects similar to those observed in the ICON7 and GOG-0218 trials.

After five phase III randomized clinical trials on combination therapy including bevacizumab and chemotherapy for ovarian cancer, ${ }^{5,6,16-18}$ the United States Food and Drug Administration (US FDA) has approved bevacizumab for all lines of ovarian cancer management. Based on results from the AU-
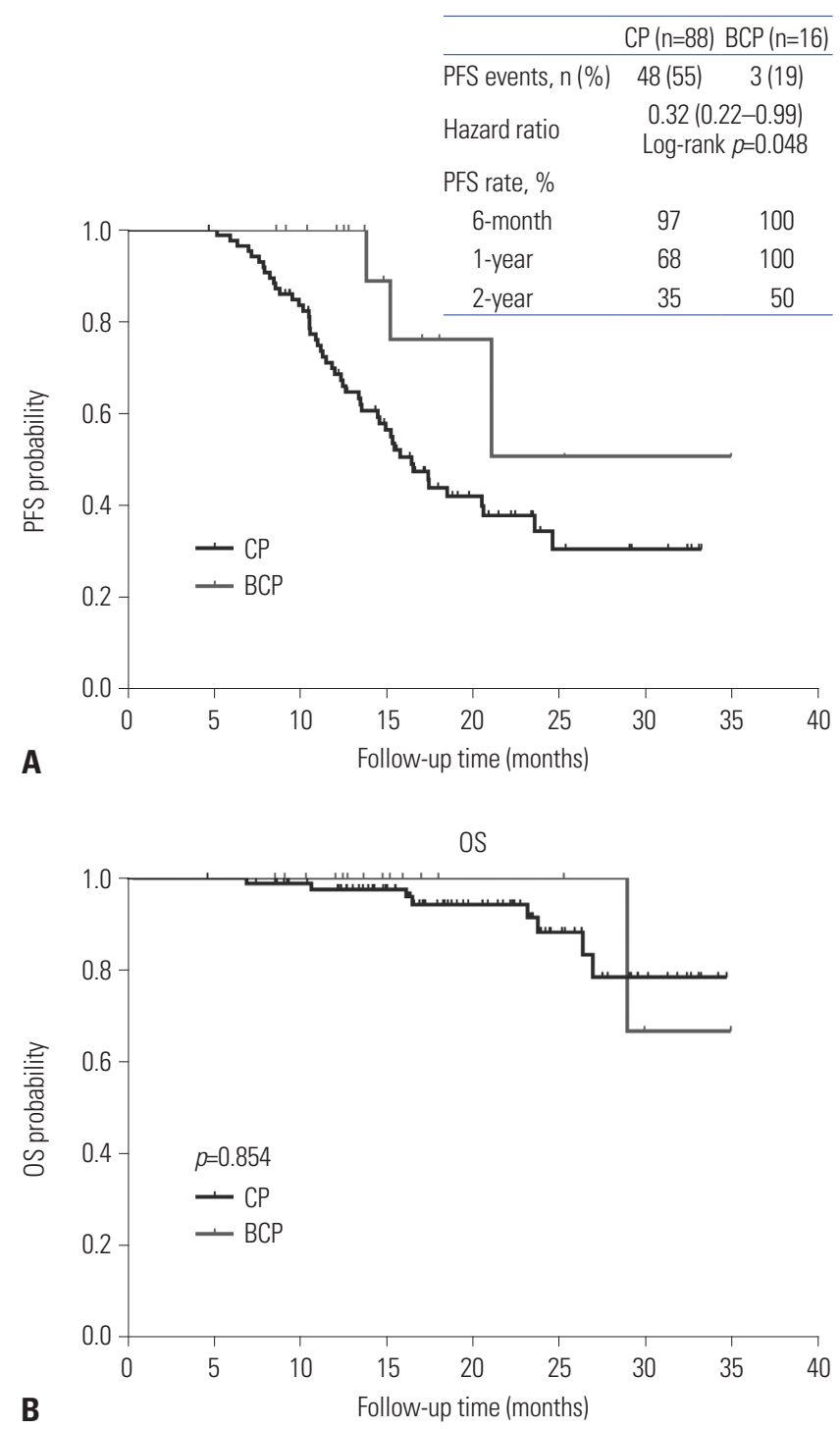

Fig. 2. Kaplan-Meier curves of progression-free survival (PFS) (A) and overall survival (OS) (B) in patients treated with standard carboplatin-paclitaxel chemotherapy $(C P, n=88)$ and bevacizumab-containing chemotherapy $(B C P, n=16)$ regimens.

RELIA, OSEAN, and GOG-213 trials, ${ }^{16-18}$ the US FDA approved bevacizumab-combined chemotherapy for recurrent ovarian cancer, ${ }^{16}$ and after the ICON-7 and GOG-218 trials, ${ }^{5,6}$ bevacizumab was approved for naïve primary ovarian cancer. Standard chemotherapy with bevacizumab in patients with newly diagnosed ovarian cancer did not result in improvements in $\mathrm{OS} ;{ }^{19}$ however, improved PFS was reported by both the ICON-7 and GOG-218 trials.

For patients with advanced ovarian cancer and with a high perioperative risk or a low likelihood of achieving optimal cytoreduction, NAC-IDS strategies are alternative treatment options. After the ICON-7 and GOG-218 trials, studies were conducted on the incorporation of bevacizumab in NAC-IDS. A subgroup analysis of the MITO-16A-MaNGO OV2A phase IV trial showed that adding bevacizumab to NAC did not impede 
IDS and resulted in a similar rate of perioperative complications, compared to conventional NAC-IDS. ${ }^{20}$ The ANTHALYA trial, a French multicenter non-comparative randomized phase II study, showed that bevacizumab could be safely added to preoperative NAC and lead to higher complete resection rates after IDS. More recently, the GEICO 1250 trial, a randomized phase II trial on bevacizumab-containing NAC, demonstrated that bevacizumab did not improve complete macroscopic response rates or PFS, but did improve surgical operability without increased toxicity. ${ }^{21}$ The current study showed that bevacizumab improved PFS, but did not show any improvement in OS. Because the follow-up period was short, more data maturity period will be needed. As for safety, the current study had one case of colonic perforation during NAC in BCP group. After emergency operation for colonic perforation, the patient recovered and then received IDS and the rest of the adjuvant chemotherapy. According to a multicenter, observational study in a real-world clinical study to evaluate the effectiveness of bevacizumab treatment based on AURELIA (KGOG 3041; REBE$\mathrm{CA}$ ), grade $\geq 3$ gastrointestinal perforation occurred only in $1.3 \%$ of the safety analysis population. ${ }^{22}$ In addition, although REBECA study was for recurrent ovarian cancer, grade $\geq 3$ adverse events were acceptable in light of real-world experience. Accordingly, we suggest that bevacizumab can generally be safely added to preoperative NAC, although careful use will be required due to the potential for serious complications, such as bowel perforation.

The limitations of current study are that it was retrospective and dependent on medical records in addition to being based in a single institution. Therefore, the sample size was relatively small, and the follow-up period was not sufficiently long to calculate OS.

In conclusion, bevacizumab-containing NAC might be safe and provide longer PFS than chemotherapy alone in patients with advanced ovarian cancer. However, further study is necessary to investigate the impact of bevacizumab-containing NAC on OS.

\section{ACKNOWLEDGEMENTS}

This study was supported by a faculty research grant of Yonsei University College of Medicine (6-2018-0169).

\section{AUTHOR CONTRIBUTIONS}

Conceptualization: Jung-Yun Lee. Data curation: Junsik Park. Formal analysis: Junsik Park. Funding acquisition: Jung-Yun Lee. Investigation: Junsik Park. Methodology: Junsik Park and Jung-Yun Lee. Project administration: Jung-Yun Lee. Resources: Kyung Jin Eoh, Eun Ji Nam, Sunghoon Kim, Sang Wun Kim, Young Tae Kim, and Jung-Yun Lee. Software: Junsik Park. Supervision: Sunghoon Kim. Validation: Young Tae Kim. Visualization: Junsik Park. Writing_original draft: Junsik Park. Writing_review \& editing: Jung-Yun Lee. Approval of final manuscript: all authors.

\section{ORCID iDs}

Junsik Park Kyung Jin Eoh Eun Ji Nam Sunghoon Kim Sang Wun Kim Young Tae Kim Jung-Yun Lee

https://orcid.org/0000-0003-4094-2097 https://orcid.org/0000-0002-1684-2267 https://orcid.org/0000-0003-0189-3560 https://orcid.org/0000-0002-1645-7473 https://orcid.org/0000-0002-8342-8701 https://orcid.org/0000-0002-7347-1052 https://orcid.org/0000-0001-7948-1350

\section{REFERENCES}

1. Vergote I, Tropé CG, Amant F, Kristensen GB, Ehlen T, Johnson N, et al. Neoadjuvant chemotherapy or primary surgery in stage IIIC or IV ovarian cancer. N Engl J Med 2010;363:943-53.

2. Kehoe S, Hook J, Nankivell M, Jayson GC, Kitchener H, Lopes T, et al. Primary chemotherapy versus primary surgery for newly diagnosed advanced ovarian cancer (CHORUS): an open-label, randomised, controlled, non-inferiority trial. Lancet 2015;386:249-57.

3. Onda T, Satoh T, Saito T, Kasamatsu T, Nakanishi T, Nakamura K, et al. Comparison of treatment invasiveness between upfront debulking surgery versus interval debulking surgery following neoadjuvant chemotherapy for stage III/IV ovarian, tubal, and peritoneal cancers in a phase III randomised trial: Japan Clinical Oncology Group Study JCOG0602. Eur J Cancer 2016;64:22-31.

4. Wright AA, Bohlke K, Armstrong DK, Bookman MA, Cliby WA, Coleman RL, et al. Neoadjuvant chemotherapy for newly diagnosed, advanced ovarian cancer: Society of Gynecologic Oncology and American Society of Clinical Oncology Clinical Practice Guideline. Gynecol Oncol 2016;143:3-15.

5. Perren TJ, Swart AM, Pfisterer J, Ledermann JA, Pujade-Lauraine E, Kristensen G, et al. A phase 3 trial of bevacizumab in ovarian cancer. N Engl J Med 2011;365:2484-96.

6. Burger RA, Brady MF, Bookman MA, Fleming GF, Monk BJ, Huang $\mathrm{H}$, et al. Incorporation of bevacizumab in the primary treatment of ovarian cancer. N Engl J Med 2011;365:2473-83.

7. Rouzier R, Gouy S, Selle F, Lambaudie E, Floquet A, Fourchotte V, et al. Efficacy and safety of bevacizumab-containing neoadjuvant therapy followed by interval debulking surgery in advanced ovarian cancer: results from the ANTHALYA trial. Eur J Cancer 2017; 70:133-42.

8. Lee YJ, Lee JY, Cho MS, Nam EJ, Kim SW, Kim S, et al. Incorporation of paclitaxel-based hyperthermic intraperitoneal chemotherapy in patients with advanced-stage ovarian cancer treated with neoadjuvant chemotherapy followed by interval debulking surgery: a protocol-based pilot study. J Gynecol Oncol 2019;30:e3.

9. Fagotti A, Vizzielli G, Fanfani F, Costantini B, Ferrandina G, Gallotta $\mathrm{V}$, et al. Introduction of staging laparoscopy in the management of advanced epithelial ovarian, tubal and peritoneal cancer: impact on prognosis in a single institution experience. Gynecol Oncol 2013;131:341-6.

10. Fagotti A, Ferrandina G, Fanfani F, Garganese G, Vizzielli G, Carone V, et al. Prospective validation of a laparoscopic predictive model for optimal cytoreduction in advanced ovarian carcinoma. Am J Obstet Gynecol 2008;199:642.e1-6.

11. Chi DS, Eisenhauer EL, Zivanovic O, Sonoda Y, Abu-Rustum NR, Levine DA, et al. Improved progression-free and overall survival in advanced ovarian cancer as a result of a change in surgical paradigm. Gynecol Oncol 2009;114:26-31.

12. Wimberger P, Lehmann N, Kimmig R, Burges A, Meier W, Du Bois A, Arbeitsgemeinschaft Gynaekologische Onkologie Ovarian Cancer Study Group. Prognostic factors for complete debulking in ad- 
vanced ovarian cancer and its impact on survival. An exploratory analysis of a prospectively randomized phase III study of the Arbeitsgemeinschaft Gynaekologische Onkologie Ovarian Cancer Study Group (AGO-OVAR). Gynecol Oncol 2007;106:69-74.

13. Eisenhauer EL, Abu-Rustum NR, Sonoda Y, Levine DA, Poynor EA, Aghajanian C, et al. The addition of extensive upper abdominal surgery to achieve optimal cytoreduction improves survival in patients with stages IIIC-IV epithelial ovarian cancer. Gynecol Oncol 2006;103:1083-90.

14. Strong VE, Selby LV, Sovel M, Disa JJ, Hoskins W, Dematteo R, et al. Development and assessment of Memorial Sloan Kettering Cancer Center's Surgical Secondary Events grading system. Ann Surg Oncol 2015;22:1061-7.

15. Chi DS, Franklin CC, Levine DA, Akselrod F, Sabbatini P, Jarnagin WR, et al. Improved optimal cytoreduction rates for stages IIIC and IV epithelial ovarian, fallopian tube, and primary peritoneal cancer: a change in surgical approach. Gynecol Oncol 2004;94: 650-4.

16. Coleman RL, Brady MF, Herzog TJ, Sabbatini P, Armstrong DK, Walker JL, et al. Bevacizumab and paclitaxel-carboplatin chemotherapy and secondary cytoreduction in recurrent, platinum-sensitive ovarian cancer (NRG Oncology/Gynecologic Oncology Group study GOG-0213): a multicentre, open-label, randomised, phase 3 trial. Lancet Oncol 2017;18:779-91.

17. Aghajanian C, Blank SV, Goff BA, Judson PL, Teneriello MG, Husain A, et al. OCEANS: a randomized, double-blind, placebo-controlled phase III trial of chemotherapy with or without bevacizum- ab in patients with platinum-sensitive recurrent epithelial ovarian, primary peritoneal, or fallopian tube cancer. J Clin Oncol 2012;30: 2039-45.

18. Pujade-Lauraine E, Hilpert F, Weber B, Reuss A, Poveda A, Kristensen $\mathrm{G}$, et al. Bevacizumab combined with chemotherapy for platinum-resistant recurrent ovarian cancer: the AURELIA openlabel randomized phase III trial. J Clin Oncol 2014;32:1302-8.

19. Oza AM, Cook AD, Pfisterer J, Embleton A, Ledermann JA, PujadeLauraine E, et al. Standard chemotherapy with or without bevacizumab for women with newly diagnosed ovarian cancer (ICON7): overall survival results of a phase 3 randomised trial. Lancet Oncol 2015; 16:928-36.

20. Daniele G, Lorusso D, Scambia G, Cecere SC, Nicoletto MO, Breda E, et al. Feasibility and outcome of interval debulking surgery (IDS) after carboplatin-paclitaxel-bevacizumab (CPB): a subgroup analysis of the MITO-16A-MaNGO OV2A phase 4 trial. Gynecol Oncol 2017;144:256-9.

21. Garcia Garcia Y, de Juan Ferré A, Mendiola C, Barretina-Ginesta MP, Gaba Garcia L, Santaballa Bertrán A, et al. Efficacy and safety results from GEICO 1205, a randomized phase II trial of neoadjuvant chemotherapy with or without bevacizumab for advanced epithelial ovarian cancer. Int J Gynecol Cancer 2019;29:1050-6.

22. Lee JY, Park JY, Park SY, Lee JW, Kim JW, Kim YB, et al. Real-world effectiveness of bevacizumab based on AURELIA in platinum-resistant recurrent ovarian cancer (REBECA): a Korean Gynecologic Oncology Group study (KGOG 3041). Gynecol Oncol 2019;152: 61-7. 